\title{
Strange nucleon form factors on $2+1$ flavor anisotropic wilson clover lattices
}

\author{
Michael Cheng*, Richard Brower, Claudio Rebbi, Oliver Witzel \\ Center for Computational Science, Boston University \\ Boston, MA 02215 USA
}

Ronald Babich, Michael A. Clark

NVIDIA Corporation

Santa Clara, CA USA

\section{Saul D. Cohen}

Department of Physics, University of Washington

Seattle, WA USA

\section{George T. Fleming}

Department of Physics, Yale University

New Haven, CT 06520 USA

\section{Joel Giedt}

Department of Physics, Renselaer Polytechnic Institute

Troy, NY USA

\section{James C. Osborn}

Argonne Leadership Computing Facility, Argonne National Laboratory

Argonne, IL 60439 USA

\section{David Schaich}

Department of Physics, University of Colorado

Boulder, CO USA

We present an update of our calculation of the strange quark contribution to the electromagnetic, axial, and scalar nucleon form factors. These calculations are performed on $2+1$ flavor, $24^{3} \times 128$ anisotropic wilson clover lattices with spatial lattice spacing $a_{s} \approx 0.12 \mathrm{fm}$ and anisotropy $\xi=3.5$. We use two different ensembles with light sea quark masses corresponding to $m_{\pi}=220,390 \mathrm{MeV}$.

The 30 International Symposium on Lattice Field Theory - Lattice 2012,

June 24-29, 2012

Cairns, Australia

\footnotetext{
${ }^{*}$ Speaker.
} 


\section{Introduction}

As the lightest non-valence quark in the nucleon, probing strange quark nucleon form factors gives insight into the non-perturbative contributions of the sea quarks to nucleon structure. There is a vigorous experimental program designed to measure the strange quark electric and magnetic form factors, $G_{E}^{s}\left(Q^{2}\right)$ and $G_{M}^{s}\left(Q^{2}\right)$. The strange axial form factor, $G_{A}^{s}\left(Q^{2}\right)$ allows one to access the contribution of the strange quark to nucleon spin. Also of interest is the strange scalar form factor, $G_{S}^{s}\left(Q^{2}\right)$. At zero momentum, this form factor gives the fraction of the nucleon mass contributed by the strange sea quarks.

$$
f_{T_{s}}=\frac{m_{s} G_{S}^{s}\left(Q^{2}=0\right)}{m_{N}}=\frac{m_{s}\langle N|\bar{s} s| N\rangle}{m_{N}} .
$$

Unfortunately, the strange scalar form factor is very difficult to measure experimentally, so Lattice QCD is a vital theoretical tool in understanding this aspect of nucleon structure. In addition, the scalar form factor is also of particular interest as it may make a significant contribution to the scattering cross sections of dark matter particles with nuclei. In many models of dark matter, the leading contribution to this cross section is via Higgs exchange, where the coupling to the nuclei is directly proportional to the sum of the scalar quark form factors [1]. Since the Yukawa couplings of the Higgs to the light quarks is quite small, while the contribution of the heavier sea quarks in the nucleon is quite suppressed, the strange quark form factor may give the dominant contribution to this process. As there are many current experiments underway that are attempting to detect dark matter via their interaction with nuclei, the need to understand the strange scalar form factor is made more vital [2].

Recently, there has been great interest in the lattice community in calculating this quantity $[3,4,5,6,7,8,9,10]$. The two most popular methods for calculating this quantity are via the Feynman-Hellman theorem, and via direct computation of the disconnected matrix elements. Here, we present some preliminary results on our calculation of the strange scalar form factor with a $2+1$ flavor anisotropic Wilson clover lattice action using direct computation of the disconnected scalar three-point function in the nucleon.

\section{Method}

The disconnected, scalar three-point function is obtained by correlating the vacuum-subtracted scalar current,

$$
j_{S}\left(\vec{x}^{\prime}, t^{\prime}\right)=\bar{s} s\left(\vec{x}^{\prime}, t^{\prime}\right)-\left\langle\bar{s} s\left(\vec{x}^{\prime}, t^{\prime}\right)\right\rangle,
$$

with the standard, zero-momentum nucleon two-point function,

$$
G^{(2)}\left(t_{f}, t_{i} ; \vec{q}\right)=\left(1+\gamma_{4}\right)^{\alpha \beta} \sum_{\vec{x}} e^{i \vec{q} \cdot \vec{x}}\left\langle N^{\beta}\left(\vec{x}, t_{f}\right) \bar{N}^{\alpha}\left(\overrightarrow{0}, t_{i}\right)\right\rangle,
$$

where $t_{f}$ and $t_{i}$ are the sink and source timeslices and $\vec{q}$ is the momentum of the nucleon.

This leads to the following expression for the three-point function:

$$
G_{S}^{(3)}\left(t_{f}, t^{\prime}, t_{i} ; \vec{q}\right)=\left(1+\gamma_{4}\right)^{\alpha \beta} \sum_{\vec{x}, \vec{x}^{\prime}} e^{i \vec{q} \cdot \vec{x}}\left\langle N^{\beta}\left(\vec{x}, t_{f}\right)\left|j_{S}\left(\vec{x}^{\prime}, t^{\prime}\right)\right| \bar{N}^{\alpha}\left(\overrightarrow{0}, t_{i}\right)\right\rangle,
$$




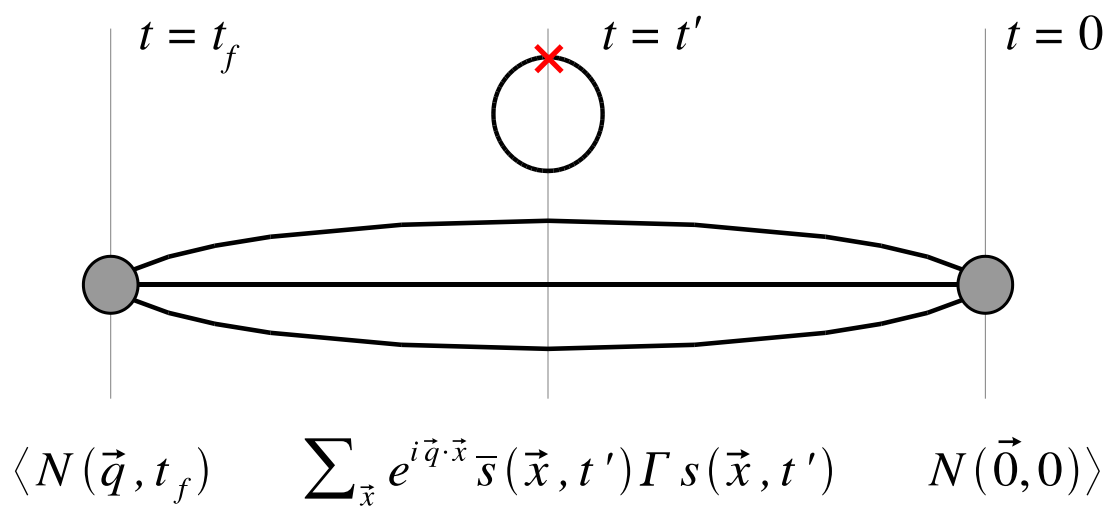

Figure 1: Schematic illustration of the evaluation of the disconnected three-point function.

$t^{\prime}$ is the timeslice for the current insertion, and now $\vec{q}$ denotes the momentum injected at the insertion. Figure 1 is a schematic illustration of the calculation of the disconnected three-point function.

The spectral decomposition of the two-point function is:

$$
G^{(2)}\left(t_{f}, t_{i} ; \vec{q}\right)=\sum_{n} 2\left(1+\frac{m_{n}}{E_{n}(\vec{q})}\right) Z_{n}^{2}(\vec{q}) e^{-E_{n}(\vec{q})\left(t_{f}-t_{i}\right)},
$$

where $\mathrm{n}$ indexes the different excited states and $Z_{n}(\vec{q})$ is the overlap of the creation operator with the state.

Similarly, for the three-point function we have:

$$
G_{S}^{(3)}\left(t_{f}, t^{\prime}, t_{i} ; \vec{q}\right)=\sum_{m, n} j_{n m}(\vec{q}) e^{-m_{n}\left(t_{f}-t^{\prime}\right)} e^{-E_{m}(\vec{q})\left(t^{\prime}-t_{0}\right)}
$$

where the matrix elements $j_{n m}(\vec{q})$ can be expressed in terms of the various form factors. In particular, the ground state matrix element can be simply related to the strange scalar form factor, $G_{s}^{S}\left(Q^{2}\right)$.

$$
j_{11}(\vec{q})=2\left(1+\frac{m_{1}}{E_{1}(\vec{q})}\right) Z_{1}(\overrightarrow{0}) Z_{1}(\vec{q}) G_{S}^{s}\left(Q^{2}\right)
$$

Thus, in the limit where the separations between the source, insertion, and sink all become large, the excited state contributions are suppressed, so that the desired form factor (at zero momentum) is given just by the ratio of correlation functions:

$$
G_{S}^{s}\left(Q^{2}=0\right)=\frac{G^{(3)}\left(t_{f}, t^{\prime}, t_{i} ; \overrightarrow{0}\right)}{G^{(2)}\left(t_{f}, t_{i} ; \overrightarrow{0}\right)}
$$

\section{Simulation Details}

We use two ensembles of anisotropic, clover-improved Wilson, stout-smeared lattices produced by the Hadron Spectrum Collaboration [11]. These lattices have a volume of $24^{3} \times 128$ lattice sites, with spatial lattice spacing $a_{s}=0.12 \mathrm{fm}$, temporal lattice spacing $a_{t}=0.035 \mathrm{fm}$, and 

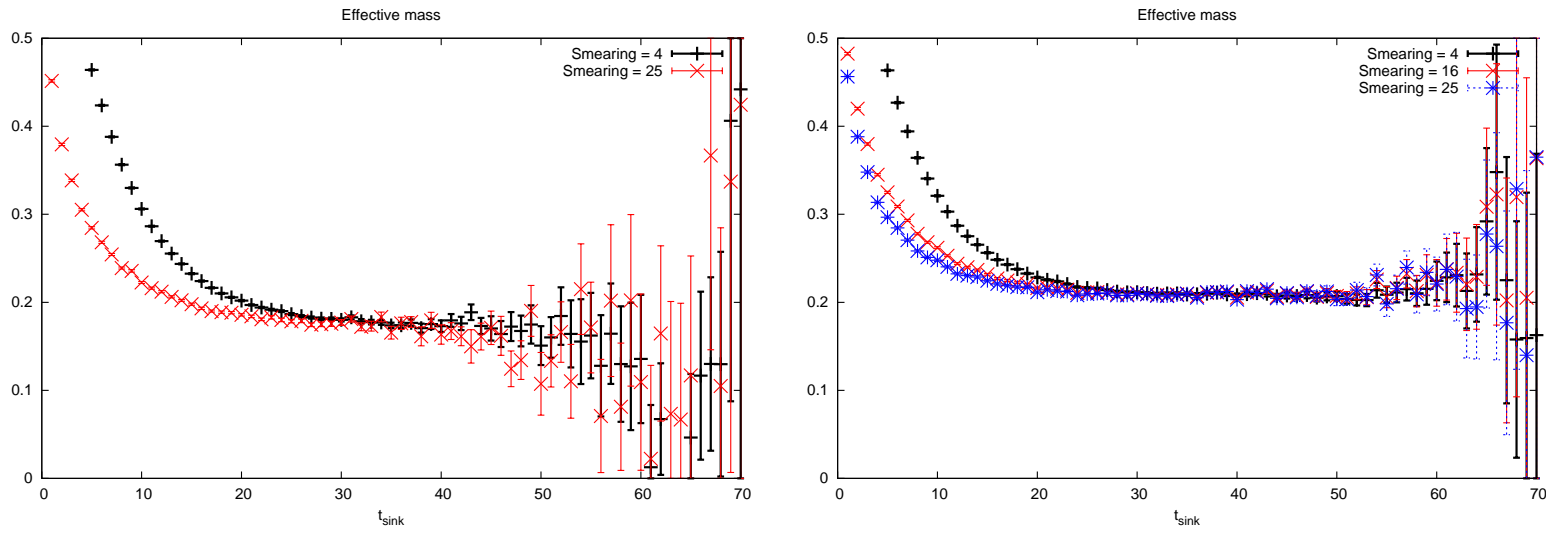

Figure 2: Effective masses from the nucleon two-point function. On the left, the effective mass for the $m_{\pi}=220 \mathrm{MeV}$ ensemble with 4 and 25 smearing steps on the source and sink. On the right, the effective mass for the $m_{\pi}=390 \mathrm{MeV}$ ensemble with 4, 16, and 25 smearing steps.

anisotropy $\xi=a_{s} / a_{t}=3.5$. Both ensembles have strange quark mass $a_{t} m_{s}=-0.0743$, but different light quark masses, $a_{t} m_{l}=-0.0860,-0.0840$. This corresponds to pion masses of $m_{\pi} \approx 220,390$ $\mathrm{MeV}$. The results that we show come from 580 configurations on the lighter pion mass ensemble and 416 configurations on the heavier pion mass ensemble, with configurations separated by 20 molecular dynamics time units.

To construct the two-point and three-point functions, we require the light quark propagator on these lattices. Because the three-point function requires the correlation between a disconnected current on the insertion timeslice with the nucleon two-point correlator, we need a large number of two-point correlators in order to extract a reasonable signal. On each configuration we place sources on every 4 timeslices. We use Gaussian-smeared point sources, where the source $\psi^{n}(x)$ after $\mathrm{n}$ smearing iterations is given by:

$$
\psi^{n}(x)=\frac{1}{1+6 r} \psi^{n-1}(x)+\frac{r}{1+6 r} \sum_{\mu=1,2,3}\left(U_{\mu}(x) \psi^{n-1}(x+\mu)+U_{\mu}^{\dagger}(x-\mu) \psi^{n-1}(x-\mu)\right),
$$

with smearing coefficient $r=1.0$. For the heavier mass ensemble, we employ sources with $n=$ $4,16,25$ smearing iterations, while for the lighter mass ensemble $n=4,25$ smearing steps are used, for a total of 96 light quark propagators per configuration for the former ensemble and 64 propagators per configuration for the latter. For each propagator, we use the same smearing at the sink as we do at the source. In addition, we effectively double our statistics by calculating also the backward-propagating correlator by replacing the $\left(1+\gamma_{4}\right)$ projection in Eq. 2.2 with $\left(1-\gamma_{4}\right)$. Thus, for the lighter mass ensemble, we measure a total of $416 \times 64=26624$ quark propagators and $580 \times 96=55680$ propagators for the heavier mass ensemble.

To compute the disconnected scalar current, we use diluted, stochastic U(1) sources to estimate the disconnected current insertion. On each configuration, we use dilution blocks of $4^{3} \times 16$, where the source is only non-zero on one site in each dilution block. By diluting the source locations, the gauge-variant noise coming from the off-diagonal matrix elements is reduced, suppressed by an exponential fall-off with the pseudoscalar meson mass. Thus, our calculation of the disconnected current insertion requires 12288 Dirac inversions per configuration, but gives a good estimate for the disconnected all-to-all propagator. 

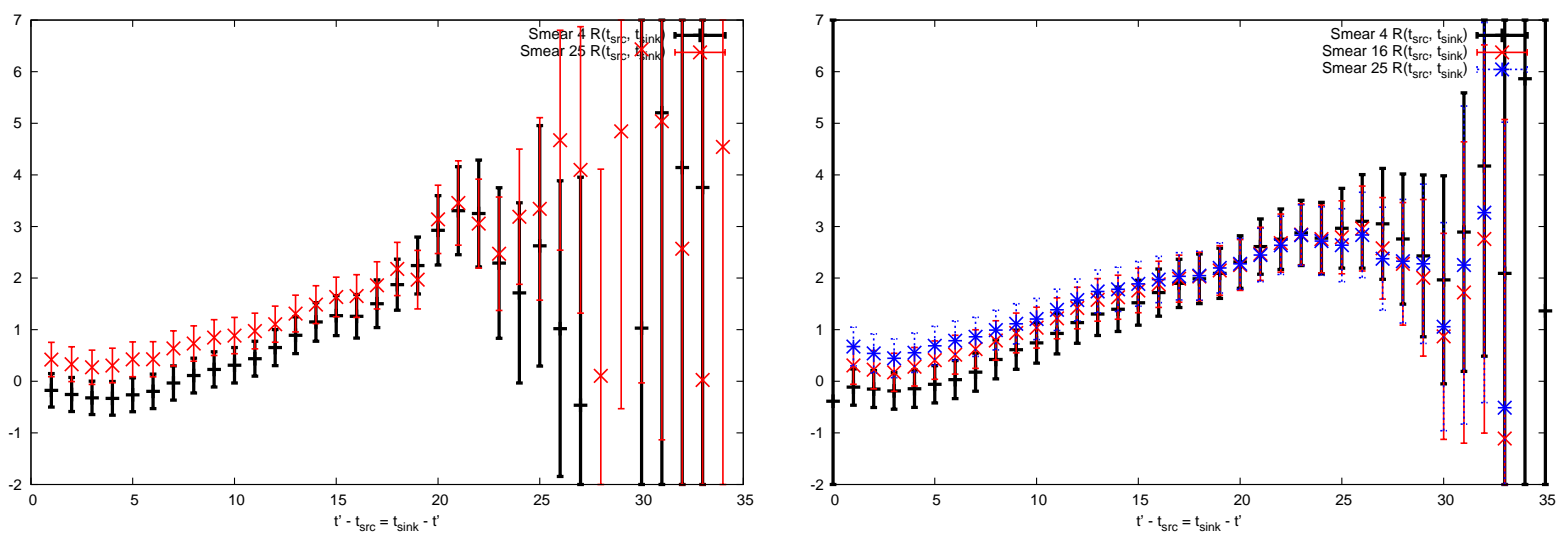

Figure 3: Ratio of the three-point function to the two-point function, $G^{(3)}\left(t_{f}, t^{\prime}, t_{i} ; \overrightarrow{0}\right) / G^{(2)}\left(t_{f}, t_{i} ; \overrightarrow{0}\right)$ for the insertion timeslice positioned halfway between the source and sink, $t_{f}-t^{\prime}=t^{\prime}-t_{i}$, as a function of this separation. The lighter pion mass ensemble is on the left, and the heavier mass ensemble is on the right.

\section{Results}

Because we have done a large number of measurements for the two-point correlation functions, we are able to make a fairly precise determination of the nucleon mass on these lattices. Figure 2 shows the nucleon effective mass for the two ensembles, for the different source and sink smearings employed.

As can be seen, the smearing of the operators tends to improve the overlap of the two-point functions with the nucleon ground state, reducing the excited state contamination at the cost of slightly increased statistical uncertainty. For the heavier mass ensemble single and double exponential fits give a robust value for the nucleon mass of $a_{t} m_{N}=0.209(1)$. However, for the lighter mass ensemble, the excited state contamination is more severe, so there is more systematic error in the choice of fit range, giving a nucleon mass of $a_{t} m_{N}=0.18(1)$.

In order to determine the desired matrix element, we need to examine the ratio of the threepoint correlator to two-point correlator given in eqn. 2.7 in the limit where all the separations between the source, insertion, and sink timeslices become large. By using diluted stochastic sources, as discussed in sec. 3, we have been able to calculate the three-point correlator with insertions at every timeslice. Thus, we are able to examine the effect of placing the insertion at any place we choose between the source and sink.

Figure 3 shows this ratio when we place the insertion midway between the source and the sink. Considering the effective mass plots in fig. 2 it is perhaps not surprising that we do not see a plateau in this ratio until the separation becomes $\sim 20$ timeslices. For both the lighter and heavier mass ensembles this puts the signal for the ground state matrix element on the edge of the regime where the statistical noise begins to wash out the signal. As can be seen, smearing of the nucleon correlator does seem to reduce the excited-state contamination, but this effect is not particularly significant.

Another interesting feature in fig. 3 is that the value for the ratio at small separations approach the plateau from below, in opposition to what is seen in the usual effective mass plot, where the ground-state mass plateau is approached from above. This seems to indicate that the dominant 

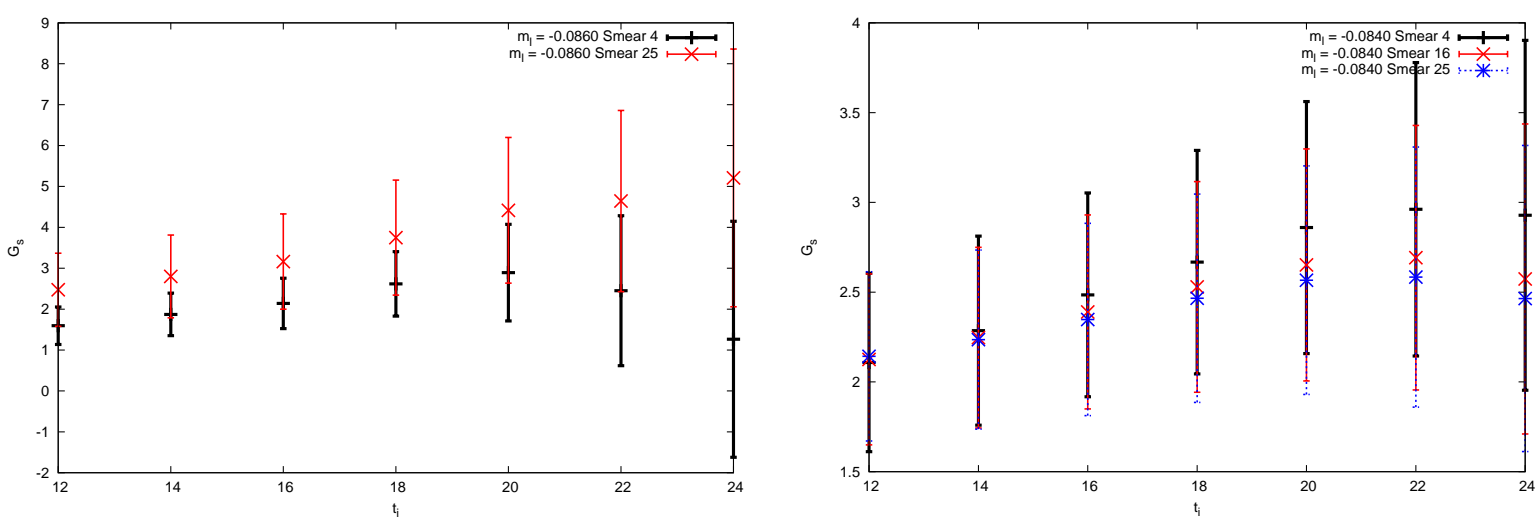

Figure 4: Fit results for the bare disconnected scalar matrix element, $G_{S}$ as a function of the minimum separation between the insertion timeslice and the source or sink location. The maximum separation included for all fits is $t_{\max }=30$ timeslices.

excited-state matrix elements enter with opposite sign compared to the ground state. Since this results in cancellation between the excited state and ground state at early times, it is difficult to apply something like a multiple exponential fit to extract the desired matrix element.

However, it is encouraging that the statistical error uncertainties shown in fig. 3 are reasonably small ( $40 \%$ for the heavier mass ensemble and $20 \%$ for the the lighter mass ensemble), yielding a value for $G_{S}^{s}\left(Q^{2}=0\right)$ that is non-zero within statistical error. Figure 4 shows the fit results for the bare strange scalar matrix element as a function the minimum separation $t_{\min }$ between the source, sink, and insertion included in the fit. In all cases, the maximum separation included is $t_{\max }=30$. One encouraging feature of fig. 4 is that the fitted result for $G_{S}^{s}\left(Q^{2}=0\right)$ do not seem to vary outside of statistical error if one includes only sufficiently large separations.

Table 1 shows the fit results with $t_{\min }=20$ and $t_{\max }=30$. As can be seen from the tabulated values, the different smearing also give results that are consistent within statistical error. The values for the heavier and lighter mass ensemble also overlap, so it is difficult to see from this data the light quark mass dependence of this quantity.

\begin{tabular}{c|cc} 
& \multicolumn{2}{|c}{$G_{S}^{s}\left(Q^{2}=0\right)$} \\
Smearing Steps & $a_{t} m_{l}=-0.0860$ & $a_{t} m_{l}=-0.0840$ \\
\hline 4 & $4.4(1.8)$ & $2.9(7)$ \\
16 & - & $2.7(6)$ \\
25 & $2.9(1.2)$ & $2.6(6)$
\end{tabular}

Table 1: Bare strange scalar matrix element $G_{S}^{s}\left(Q^{2}=0\right)$ for the light and heavy ensemble as a function of the source and sink smearing. All values extracted with fit range $t_{\min }=20$ and $t_{\max }=30$.

\section{Conclusions and Future Goals}

If one takes the preliminary result of our calculation as $\langle N|\bar{s} s| N\rangle \approx 3.0$, combined with $a_{t}\left(m_{s}-\right.$ $\left.m_{\text {crit }}\right) \approx 0.013$ and $a_{t} m_{N} \approx 0.2$ for the lattices we have used, one one obtains a value of $f_{T_{s}} \approx$ 
0.2 , which is significantly larger than the results of other lattice calculation, which give $f_{T_{s}}<0.1$ $[3,6,7,8]$. The reason for this is that the above calculation uses the value of the bare scalar matrix element. Because we use Wilson fermions, the explicit breaking of chiral symmetry results in different renormalization factors for the singlet and non-singlet mass terms [7, 12]. Therefore, in order to get results which can reliably be compared to other calculations, this renormalization procedure must be carried out.

To do this, we intend to calculate the connected and disconnected scalar form factors for the light quarks as well. By summing the light and strange contributions to the form factor on our 2+1 flavor lattices, we can obtain an RG-invariant quantity which encapsulates the coupling of the Higgs boson to the three lightest quarks in the nucleon.

It is also clear from our data that there is significant excited state contamination in our results. We will explore methods, such as using a variational basis with different source/sink smearings to better extract the ground state contribution.

\section{References}

[1] A. Bottino, F. Donato, N. Fornengo, and S. Scopel, Astropart.Phys. 18, 205 (2002), hep-ph/0111229.

[2] J. Giedt, A. W. Thomas, and R. D. Young, Phys.Rev.Lett. 103, 201802 (2009), 0907.4177.

[3] R. Young and A. Thomas, Phys.Rev. D81, 014503 (2010), 0901.3310.

[4] H. Ohki, H. Fukaya, S. Hashimoto, T. Kaneko, H. Matsufuru, et al., Phys.Rev. D78, 054502 (2008), 0806.4744 .

[5] H. Ohki, S. Aoki, H. Fukaya, S. Hashimoto, T. Kaneko, et al., PoS LAT2009, 124 (2009), 0910.3271.

[6] W. Freeman and D. Toussaint (MILC Collaboration) (2012), 1204.3866.

[7] G. S. Bali et al. (QCDSF Collaboration), Phys.Rev. D85, 054502 (2012), 1111.1600.

[8] S. Dinter et al. (ETM Collaboration), JHEP 1208, 037 (2012), 1202.1480.

[9] R. Babich, R. C. Brower, M. A. Clark, G. T. Fleming, J. C. Osborn, et al., Phys.Rev. D85, 054510 (2012), 1012.0562.

[10] A. Thomas, P. Shanahan, and R. Young, Nuovo Cim. C035N04, 3 (2012), 1202.6407.

[11] H.-W. Lin et al. (Hadron Spectrum Collaboration), Phys.Rev. D79, 034502 (2009), 0810.3588.

[12] C. Michael, C. McNeile, and D. Hepburn (UKQCD Collaboration), Nucl.Phys.Proc.Suppl. 106, 293 (2002), hep-lat/0109028. 